\title{
Non à la clause du besoin qui complique l'avenir des jeunes médecins
}

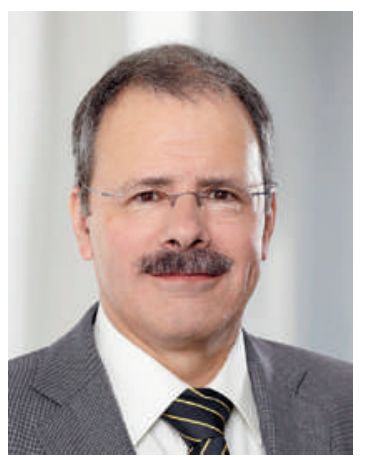

Le «stop and go» est rarement une bonne solution. Il se pourrait néanmoins que les Chambres fédérales adoptent un nouveau gel des admissions pour les médecins spécialistes, applicable dès le $1^{\text {er }}$ avril 2013. D'abord pour une durée de trois ans; mais sait-on jamais? Le précédent en a duré dix.

A première vue, on peut comprendre les raisons d'un nouveau gel des admissions. En forte hausse l'an dernier, les demandes d'autorisation de pratiquer et de numéros RCC continuent d'augmenter, ce à quoi

\section{La clause du besoin est un mauvais signal pour la relève médicale de notre pays.}

il fallait d'ailleurs s'attendre après une longue période de restriction - d'autant plus que le «go» risque d'être suivi d'un nouveau «stop». Et nul ne sait si les numéros RCC nouvellement délivrés seront effectivement utilisés, ni dans quelle mesure ils le seront - par exemple à temps plein ou à temps partiel.

Une décision ne reposant pas sur des données fiables serait irresponsable vis-à-vis des jeunes médecins, hommes et femmes, qui ont suivi une très bonne formation en Suisse. Ils se trouveraient pénalisés par une mesure dont on peut à juste titre se demander si, s'étendant sur plusieurs années, elle n'enfreint pas la liberté économique garantie par la Constitution. Réintroduire la clause du besoin serait en outre une décision déraisonnable qui enverrait un mauvais signal à une relève médicale dont la Suisse a un besoin urgent. Cela aurait également pour effet d'entraver le développement de nouveaux modèles de pratique multidisciplinaire.

Si la FMH reconnaît le besoin de réguler la répartition des médecins, elle s'élève en revanche contre des méthodes à la va-vite et un «stop and go» décidé dans la précipitation. La réalité exige au contraire une approche différenciée, flexible et cantonale: à Lugano, Bâle et Genève, nous avons par exemple trop de médecins, mais pas assez par contre dans les régions périphériques. Hors agglomérations se dessine déjà une pénurie non seulement de médecins de famille, mais également de gynécologues, de psychiatres, de chirurgiens et d'autres spécialistes.

Pour la FMH, un gel temporaire des admissions de spécialistes ne peut entrer en ligne de compte que si les quatre conditions suivantes sont mises en œuvre:

- Les médecins qui ont exercé pendant au moins cinq ans dans un hôpital reconnu pour la formation ne sont pas soumis à la clause du besoin. Comme ils connaissent déjà le système de santé helvétique, ils sont à même de fournir un travail efficace et de haute qualité.

- Après avoir obtenu leur autorisation de pratiquer, les médecins ont un an pour ouvrir leur propre cabinet.

- Les médecins hospitaliers doivent être inclus dans la clause du besoin. Il est inacceptable de prononcer le gel de cabinets médicaux tout en continuant de développer le secteur ambulatoire des hôpitaux.

- Les médecins au bénéfice d'un titre postgrade «médecin praticien» doivent également être soumis à la clause du besoin. Les détenteurs de ce titre ont soit une formation postgraduée très courte ou sont des spécialistes venus de l'étranger qui n'affichent pas leur titre de manière transparente.

\section{Le «stop and go» n'est pas une option pour la FMH.}

Le «stop and go» n'est pas, en l'occurrence, une bonne façon d'agir. Le pilotage du système de santé doit tenir compte de la réalité et toujours avoir en vue - il va de soi - le bien-être des patientes et des patients.

Dr Jürg Schlup, président de la FMH 